\title{
By-product of the V1162 Ori multisite campaign: the new $\delta$ Scuti star GSC 477800324
}

\author{
Lampens, P. ${ }^{1}$, Van Cauteren, P. ${ }^{2,5}$, Niarchos, P. ${ }^{3}$, Gazeas, K. ${ }^{3}$, \\ Manimanis, V. ${ }^{3}$, Arentoft, T. ${ }^{1,4}$, Wils, P. ${ }^{5}$, Bruch, A. ${ }^{6}$, Garrido, R. ${ }^{7}$, \\ Shobbrook, R. ${ }^{8}$ \\ ${ }^{1}$ Koninklijke Sterrenwacht van België, Ringlaan 3, 1180 Brussels, Belgium \\ ${ }^{2}$ Beersel Hills Observatory (BHO), Laarheidestraat, 3, 1650 Beersel, Belgium \\ ${ }^{3}$ Section of Astrophysics, Astronomy and Mechanics, University of Athens, 15783 \\ Zografos, Athens, Greece \\ ${ }^{4}$ Vrije Universiteit Brussel, Pleinlaan 2, 1050 Brussels, Belgium \\ ${ }^{5}$ Vereniging Voor Sterrenkunde (VVS), Belgium \\ ${ }^{6}$ Laboratório Nacional de Astrofísica, C.P. 21, 37500-000, Itajubá - MG - Brazil \\ ${ }^{7}$ Instituto de Astrofísica de Andalucía, CSIC, Apdo. 3004, 18080 Granada, Spain \\ ${ }^{8}$ Research School of Astronomy and Astrophysics, Australian National University, \\ Weston Creek PO, ACT 2611, Australia
}

\section{Introduction}

Among the comparison stars used to monitor the intriguing intermediate amplitude $\delta$ Scuti V1162 Ori two new variable stars have been discovered in the same field. One of them is GSC 477800324 , for which short-period variability with an amplitude of about 0.01 mag with respect to the "official" comparison star had been reported previously (Van Cauteren and Lampens, 2000). This variability was also the cause for the first claim of multiperiodicity for V1162 Ori (Hintz et al. 1998). The colour indices obtained by Handler (2000) put the new variable star in the middle of the $\delta$ Scuti instability strip. Therefore we decided to monitor GSC 477800324 taking as much as possible advantage from the international multisite campaign on V1162 Ori itself (Arentoft et al. 2001). In various cases the effective field-of-view was too small to allow simultaneous measuring with the main target and a few participants secured data by dedicating some extra time. However, the bulge of the data discussed here comes from those smaller telescopes with a sufficiently large chip that allow to simultaneously obtain data on both $\delta$ Scuti stars (Belgium and Greece). Figure 1 shows the field with the identification of the known and the new variable stars. The $\delta$ Scuti candidate is called "New 1". The star called "New 2" is a new 
eclipsing binary with an orbital period very close to an integer multiple of one day. The present contribution reflects the contents of a poster, presented at IAU Symp. 185 (Lampens et al. 2002), in more detail and reports on the final data analysis for the new $\delta$ Scuti star.

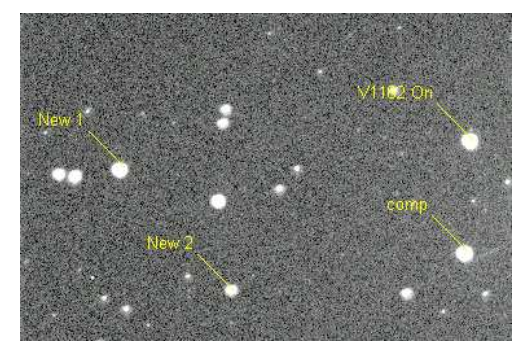

Figure 1: The field of V1162 Ori $\left(8 \times 12 \operatorname{arcmin}^{2}\right)$ and the newly detected variable stars.

\section{Data and reduction}

The observations were collected between October 1999 and March 2001. All observations used here were made using a standard V filter. Occasionally the $\mathrm{R}$ filter was used. The reduction was performed by each individual observing team. In some cases the packages MOMF (Kjeldsen and Frandsen 1992; by TA) or MIRA AP (Axiom Research Inc.; by PVC) were used. Table 1 lists the individual contributions. We mention the name of the observer, the location of the observatory, the number of measurements, an identifier for the comparison star and the mean data string length. In total we have made use of 2949 data points for the analysis of GSC $477800324(\mathrm{~V}=10.26$; $b-y=0.20)$. In principle the same comparison star as used for the V1162 Ori campaign was adopted (GSC $477800019, V=9.73$; $B-V=1.55$ ). Arentoft et al. (2001) have shown that this comparison star shows nightly zero-point shifts but only at the level of 0.01-0.02 mag such that this will not affect the frequency search in the range of interest (see below). In addition all differential data have been rescaled to the same mean difference in magnitude. The time base length of the complete data sets is 520 days. This allows a theoretical frequency resolution of $1.5 / \mathrm{T}$ or 0.0029 cycles/day. 
Table 1: Logbook of observations of GSC 4778-00324

\begin{tabular}{|cccccc|}
\hline Name & Observatory & Nr of data & $\begin{array}{c}\text { Comp.star } \\
\text { (S=GSC4778) }\end{array}$ & $\begin{array}{c}\text { Mean length } \\
\text { (days) }\end{array}$ & Remark \\
\hline Bruch & OPD & 432 & S 285 & 0.158 & C2 used \\
Garrido & Sierra Nevada & 23 & S 019 & 0.073 & \\
Lampens et al. & Hoher List & 53 & S 019 & 0.080 & \\
Niarchos et al. & Athens Univ. & 2467 & S 019 & 0.103 & \\
Shobbrook & Siding Spring & 13 & S 019 & 0.120 & \\
Sterken & ESO & 105 & S 019 & 0.093 & \\
Van Cauteren & Beersel Hills & 1054 & S 019 & 0.106 & \multirow{2}{*}{ T=520 d } \\
All & & 4147 & S 019 & 95.7 hrs & . \\
\hline
\end{tabular}

\section{Frequency analyses}

The same criteria as for the final data selection of V1162 Ori were applied also in this case (Arentoft et al. 2001). The analysis was performed in three steps: (a) on the (northern) winter 1999-2000 data set, (b) on the (northern) winter 2000-2001 data set, and (c) on the complete data set. In all cases the data points were weighted in proportion to the inverse square of the residuals on a night-to-night basis. In each set up to three frequencies could be identified. We are confident that the two most dominant frequencies are secure as they are common to each individual analysis and have signal-to-noise ratios well above 4. Figure 2 illustrates the frequency search for the complete data set. Figure $2 \mathrm{a}$ illustrates the periodogram of the original data, the subsequent frames show the periodograms after prewhitening with the most dominant frequency of the previous frame. Whereas there is no doubt about the main frequency at 12.28 cycles/day, some ambiguity arises concerning the second frequency. In our previous analysis (Lampens et al. 2002) we adopted the frequency at $16.62 \mathrm{c} / \mathrm{d}$ as the most probable second frequency since it appeared to be very slightly more dominant in the yearly analyses in which all the data points were treated equally. However, Figure $2 b$ now shows that the 1 /day alias frequency at 15.62 cycles/day is slightly stronger (the corresponding amplitude is larger by $1 \mathrm{mmag}$ ). This, together with the symmetry of the alias pattern in Figure $2 \mathrm{~b}$ ( 5 distinguishable peaks at each side of the adopted peak), causes us to identify the latter as the most probable second frequency. After simultaneous prewhitening with the two most dominant frequencies, the remaining standard deviation is $6.7 \mathrm{mmag}$. The nightly rms values of the data usually range from 3 to $10 \mathrm{mmag}$. Considering the possible contribution of slight zero-point shifts in the shortest data sets, this may reflect the overall precision of the differential data. But the periodogram in Figure $2 c$ still exhibits additional power in the range 10 15 cycles/day. Possible values for a third frequency together with the most 
dominant frequencies are summarized in Table 2, presenting the final results of the analysis. Depending on the choice for the second frequency (either 15.62 or 16.62 cycles/day) the third frequency assumes quite distinct values and its value is therefore uncertain even though it was identified with a signal-to-noise ratio larger than 4 in most cases. The ratio between the two most dominant frequencies is either $0.74(16.62 \mathrm{c} / \mathrm{d})$ or $0.79(15.62 \mathrm{c} / \mathrm{d})$.
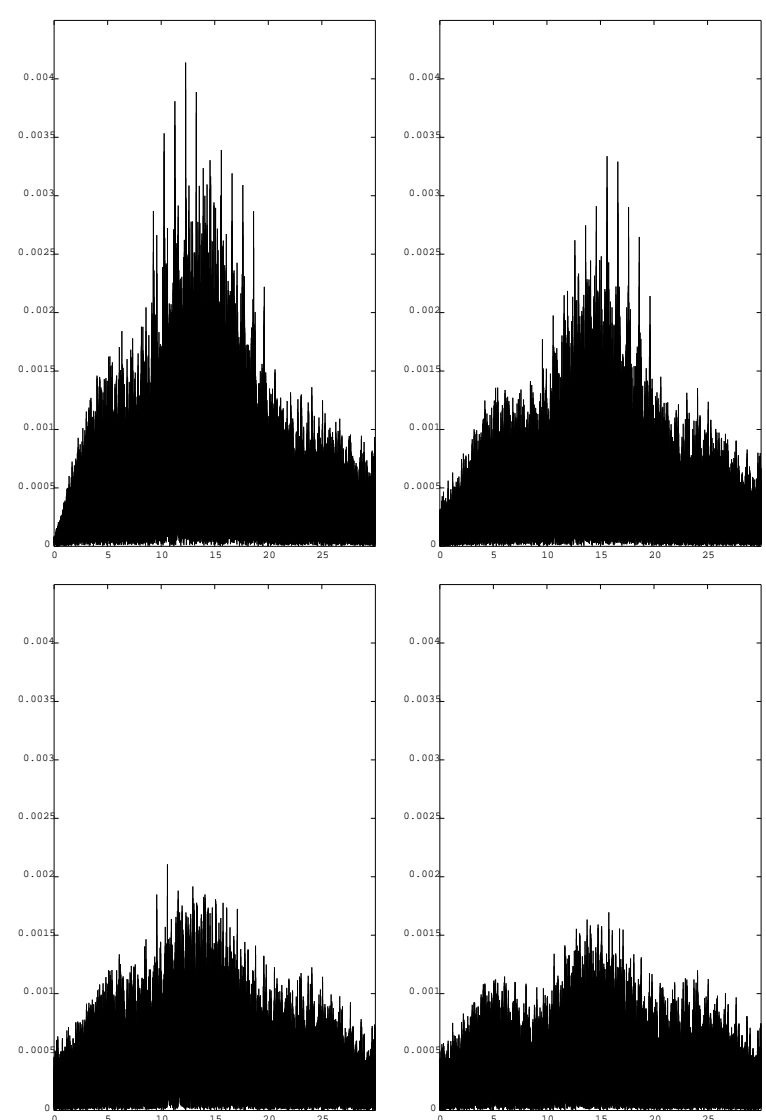

Figure 2: Periodograms of successive frequency searches (a-d: from left to right).

Figure 3 illustrates two of the associated mean light curves. The $\mathrm{V}$ amplitudes are of order of several millimags only. It must be remarked that the solutions presented here are incomplete as there exists at least one string of data for which the fit is not quite satisfactory (on JD 2451575). The best 

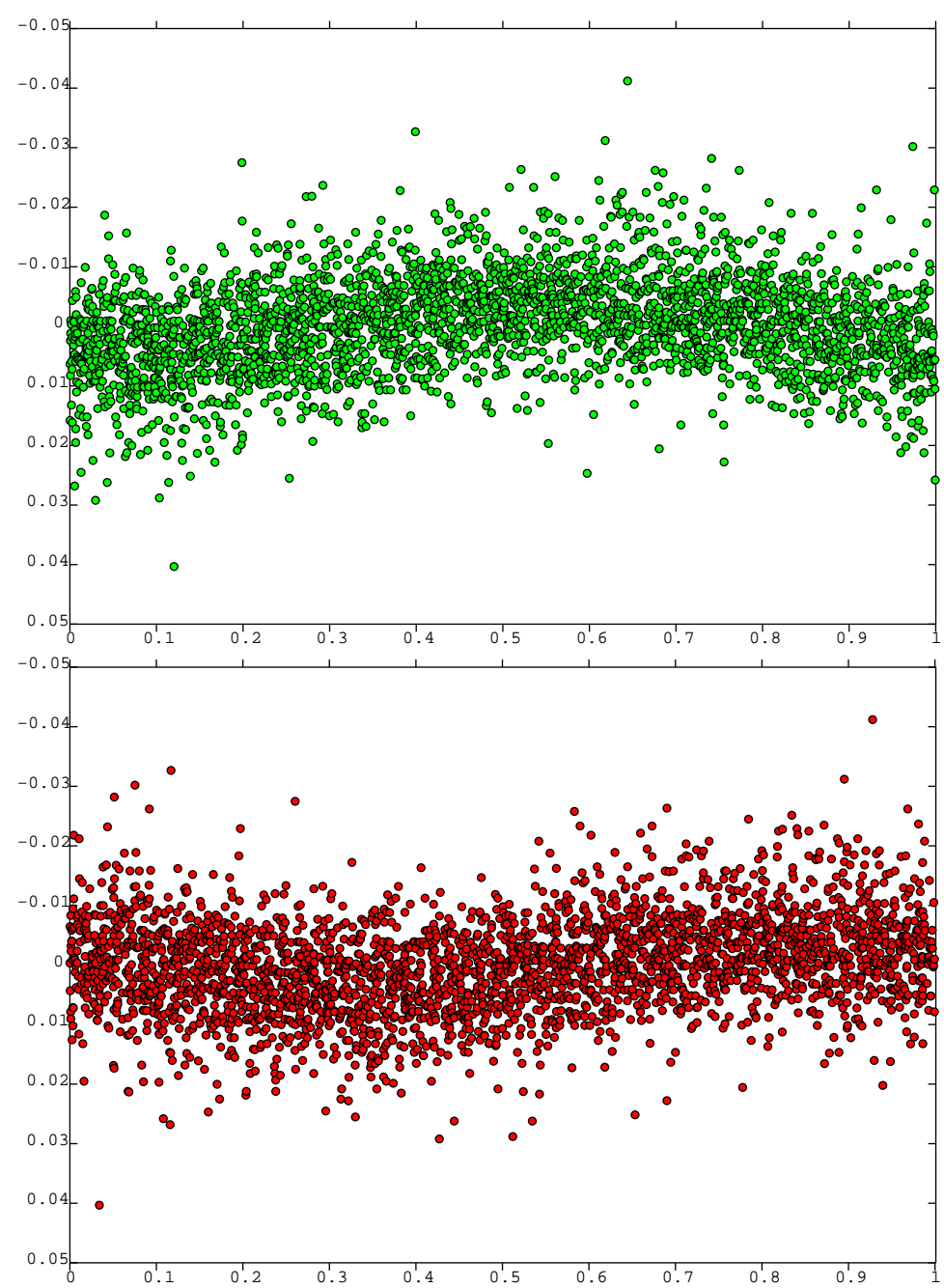

Figure 3: Phase diagrams at the frequencies of $12.28 \mathrm{c} / \mathrm{d}$ (left) and $15.62 \mathrm{c} / \mathrm{d}$ (right) after prewhitening for the other frequency.

solution in terms of largest reduction of the standard deviation is flagged (1) in Table 2. The long, continuous data set obtained in Brazil (Table 1) was used as an independent test of the proposed frequency solution. Figure 4 shows the yet unused data as well as the two-frequency fit from solution (1). We readjusted the phases in order to have a perfect match between the calculated and the 
Table 2: Frequency analyses for GSC 477800324

\begin{tabular}{|c|c|c|c|c|c|c|c|}
\hline Season & $\mathrm{Nr}$ & Ident. & $\begin{array}{c}\text { Freq. } \\
\mathrm{c} / \mathrm{d}\end{array}$ & $\underset{\mathrm{mag}}{\text { Ampl. }}$ & $\begin{array}{c}\text { Phase } \\
2 \pi \mathrm{rad}\end{array}$ & $\mathrm{S} / \mathrm{N}$ & Remark \\
\hline $1999-2000$ & 1546 & $\begin{array}{l}f_{1} \\
f_{2} \\
f_{3}\end{array}$ & $\begin{array}{l}12.2801 \\
15.6157 \\
11.7173 \\
\end{array}$ & $\begin{array}{l}0.0041 \\
0.0034 \\
0.0023\end{array}$ & $\begin{array}{l}0.960 \\
0.927 \\
0.317\end{array}$ & $\begin{array}{l}7.8 \\
5.6 \\
4.3\end{array}$ & $\begin{array}{c}\text { weighted data } \\
\text { RSS }=6.3 \mathrm{mmag} \\
\text { uncertain }\end{array}$ \\
\hline $2000-2001$ & 1403 & $\begin{array}{l}\mathrm{f}_{1} \\
\mathrm{f}_{2} \\
f_{3}\end{array}$ & $\begin{array}{l}12.2802 \\
16.6215 \\
13.2568\end{array}$ & $\begin{array}{l}0.0048 \\
0.0038 \\
0.0027\end{array}$ & $\begin{array}{l}0.671^{2} \\
0.827^{2} \\
0.367^{2}\end{array}$ & $\begin{array}{l}7.4 \\
6.9 \\
4.4 \\
\end{array}$ & $\begin{array}{c}\text { weighted data } \\
\mathrm{RSS}=6.7 \mathrm{mmag} \\
\text { uncertain }\end{array}$ \\
\hline (1) $1999-2001$ & 2949 & $\begin{array}{l}\mathrm{f}_{1} \\
\mathrm{f}_{2} \\
f_{3}\end{array}$ & $\begin{array}{l}12.2810 \\
15.6169 \\
10.5852\end{array}$ & $\begin{array}{l}0.0041 \\
0.0036 \\
0.0021\end{array}$ & $\begin{array}{l}0.136 \\
0.135 \\
0.421\end{array}$ & $\begin{array}{l}8.1 \\
6.8 \\
4.7\end{array}$ & $\begin{array}{l}\text { weighted data } \\
\text { strong alias at } 16.62 \mathrm{c} / \mathrm{d} \\
\mathrm{RSS}=6.3 \mathrm{mmag}\end{array}$ \\
\hline (2) 1999-2001 & 2949 & $\begin{array}{l}f_{1} \\
f_{2} \\
f_{3}\end{array}$ & $\begin{array}{l}12.2810 \\
16.6171 \\
12.9543\end{array}$ & $\begin{array}{l}0.0042 \\
0.0027 \\
0.0021\end{array}$ & $\begin{array}{l}0.118 \\
0.611 \\
0.802\end{array}$ & $\begin{array}{l}8.2 \\
4.8 \\
3.9\end{array}$ & $\begin{array}{l}\text { weighted data } \\
\text { strong alias at } 15.62 \mathrm{c} / \mathrm{d} \\
\mathrm{RSS}=6.5 \mathrm{mmag}\end{array}$ \\
\hline
\end{tabular}

${ }^{2}$ Phases corrected for the origin difference between data sets

observed light curve. The standard deviation of the residuals dropped by $24 \%$ to the level of $6.4 \mathrm{mmag}$. This shows that the quality of the presently available data set is probably not sufficient to permit the identification of additional frequencies with amplitudes as small as 2-3 mmag.

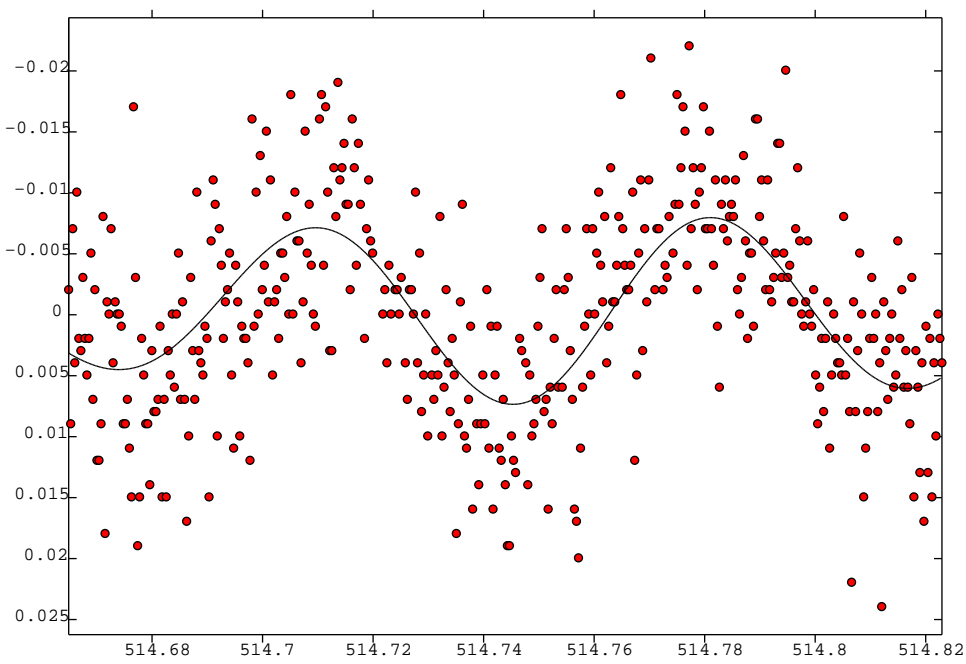

Figure 4: Observations from OPD, Brazil, and two-frequency fit (JD 2451514). 


\section{Conclusions}

A by-product of the international multisite campaign on V1162 Ori is the serendipitous discovery of a pulsating star of very small amplitude and with variable light curve among the comparison stars in the field. GSC 477800324 has the colours of a typical $\delta$ Scuti star. This star was frequently observed during the 1999-2000 and 2000-2001 seasons, mainly from Greek and Belgian sites. The final frequency analysis, based on assigning weights to the data, confirms the $\delta$ Scuti type and the presence of at least two and probably three frequencies in the range 10-15 cycles/day. $V$ amplitudes are of order 4 mmag or smaller. The proposed frequency solutions are incomplete but the presently available data set does not permit the identification of more frequencies. In itself it is remarkable that observations from non-ideal sites in the vicinity of large European cities (Athens and Brussels) allow to detect and monitor such subtle variations and can effectively contribute to the analysis of these small-amplitude multiperiodic pulsating stars.

Acknowledgments. This research was supported by project G.0265.97 of the Belgian Fund for Scientific Research (FWO) - Flanders. T. Arentoft (TA) acknowledges financial support by the Flemish Ministry for Foreign Policy, European Affairs, Science and Technology, under contract BIL 98/11/52 and by the National Research Foundation of South Africa. We thank C. Sterken for providing us with observations of the new variable star. R.R. Shobbrook, Honorary Visiting Fellow at the Australian National University, acknowledges support from Mount Stromlo and Siding Spring Observatories. P. Lampens and P. Van Cauteren (PVC) thank Prof. Dr. W. Seggewiss for granting telescope time at the Hoher List Observatory (University of Bonn, Germany). We also thank M. Sperl for updated versions of the programme Period98.

\section{References}

Arentoft, T. et al. 2001, A\&A 374, 1056

Handler, G. 2000, IBVS 4817

Hintz, E., Joner, M. \& Kim, C. 1998, PASP 110, 689

Kjeldsen, H. \& Frandsen, S. 1992, PASP 104, 413

Lampens, P. et al. 2002, poster in Proceedings of IAU Coll. 185 on Radial and Nonradial Pulsations as Probes of Stellar Physics, Leuven, Belgium, 26-31 July,

2001. ASP Conference Series, eds. C. Aerts, T. Bedding \&

J. Christensen-Dalsgaard, in press.

Van Cauteren, P. \& Lampens, P. 2000, IBVS 4849 